\title{
La internacionalización de los mapuche: entre pueblo indígena y nación
}

\author{
Pablo Barnier-Khawam \\ Sciences Po Paris, CERI/CNRS, París, Francia. \\ Email: pablo.barnierkhawam@sciencespo.fr
}

Resumen: El presente trabajo surge de la contradicción entre el creciente llamado a ser miembro del mundo global, y el desarrollo de identidades particulares que se constituyen a partir de las posibilidades que emanan de esa integración en el espacio global. Lo anterior muestra que la internacionalización, que se divide en una dinámica nacional de proyección internacional y una dinámica internacional de penetración nacional, es clave en la construcción identitaria de los mapuche. La metodología del trabajo se funda en una perspectiva comprensiva del fenómeno en base a la realización de entrevistas a diversos actores, la consulta de archivos, y una observación participante en el interior del Ministerio de Relaciones Exteriores de Chile. Los resultados de esta investigación muestran que la internacionalización de los mapuche conduce a la división de su identidad entre dos referentes de identificación que son el de "indígena” y el de "nación”, cada uno con discursos y prácticas específicas.

Palabras claves: Internacionalización, pueblo mapuche, Comité Exterior Mapuche, indígena, autodeterminación.

\section{The internationalization of the Mapuche: between indigenous people and nation}

\begin{abstract}
This work starts from the contradiction between the growing call to be a member of a global world and the development of particular identities that are constituted from the possibilities that arise from such integration in the global space. It shows that internationalization, which is divided into a national dynamic of international projection and an international dynamic of national penetration, is key in the construction of Mapuche identity. Its methodology is based on a comprehensive perspective of the phenomenon thanks to the use of several interviews with actors, the consultation of archives and a participating observation within the Ministry of Foreign Affairs of Chile. The results of this research show that the internationalization of the Mapuche leads to the division of their identity between two referents of identification that are those of "indigenous" and "nation" and that each one implies specific discourses and practices.
\end{abstract}

Keywords: Internationalization, identification referent, Mapuche people, social movement, indigenous people.

\section{A internacionalização dos mapuche: entre povos indígenas e a nação}

Resumo:Este trabalho parte da contradição entre o chamado crescente de ser um membro de um mundo global e o desenvolvimento de identidades particulares que são constituídas a partir das possibilidades que emanam dessa integração no espaço global. Isso mostra que a internacionalização, que é dividida em uma dinâmica nacional de projeção internacional e uma dinâmica internacional de penetração nacional, é fundamental na construção da identidade dos Mapuche. Sua metodologia é baseada numa perspectiva abrangente do fenômeno graças ao uso de várias entrevistas com atores, a consulta de arquivos e uma observação participante dentro do Ministério das Relações Exteriores do Chile. Os resultados desta pesquisa mostram que a internacionalização dos Mapuche leva à divisão de sua identidade entre duas referências de identificação que são as de "indígena” e de "nação" e que cada uma implica discursos e práticas específicas.

Palavras-chave: Internacionalização, referência de identificação, povo mapuche, movimento social, povo indígena. 


\section{Introducción}

Ka Mapu Mapuche Traum (Comité Exterior Mapuche, 1980), o reunión lejos de la patria, es el título de la primera reunión del Comité Exterior Mapuche (CEM), organizada en 1978 en Londres, por mapuche ${ }^{1}$ exiliados en Europa tras el golpe de Estado del 11 de septiembre1973 en Chile. Si bien este encuentro demuestra la capacidad de los mapuche para organizarse en el exterior, no es la primera vez que el pueblo mapuche entra en una dinámica de internacionalización. Ya las interacciones con los colonizadores españolestanto por la guerra, por la realización de los numerosos parlamentos (Pichinao Huenchuleo, 2013), como por el vínculo establecido entre la Federación Araucana y el Socorro Rojo Internacional² (Mallon, 2011), comprueban la participación de los mapuche en las dos dinámicas de la internacionalización: una nacional con proyección internacional y una internacional con penetración nacional (Devin, 2001).

Sin embargo, esa internacionalización hace parte dela redefinición identitaria de los mapuche, cuestión que se acentúa a partir del golpe de 1973 producto de diversos procesos que este trabajo tiene por propósito comprender. Surge así la contradicción entre la creciente llamada a ser miembro de un mundo global y, el desarrollo de identidades particulares que se constituyen a partir de las posibilidades que emanan de esa integración en el espacio global. Dicha contradicción la advierte Arjun Appadurai, al señalar:

“el desplazamiento y el exilio, la migración y el terror crean potentes vínculos con la idea de una patria (homeland) que parece más territorial que nunca. Pero es también posible detectar en varias de esas transnaciones (...) los elementos de un imaginario postnacional”(1996, pp. 176-177, traducción propia).

Los procesos de cambio identitario demuestran que la identidad mapuche contemporánea pasó por diversas etapas de definición que hicieron variar lo que entenderemos como sus referentes de identificación, o sea aquella categoría a partir de la cual se construye una identidad y que implica prácticas y discursos distintos según sus contextos, dando así a esta última un carácter dinámico y multidimensional (Cuche, 2016). De etnia a pueblo indígena y de pueblo indígena a nación, la internacionalización de los mapuche va a ser un factor esencial en la definición identitaria y, por ende, de la política del pueblo mapuche. Esta búsqueda de definición culminará en un concepto clave entre la relación del pueblo mapuche y el Estado chileno, que es el de autodeterminación, y que fue en parte introducido por la movilización internacional del pueblo mapuche. Así, nos podemos preguntar:

¿Cómo la internacionalización del pueblo mapuche participa en su redefinición identitaria, y por ello, rearticula su relación con el Estado chileno?

Para responder a esta pregunta, veremos que en un primer momento el exilio de varios militantes mapuche y la movilización internacional que le sigue contribuyeron en la redefinición identitaria de los mapuche. Esta última será, en un segundo momento, el origen de la redefinición de la relación entre el Estado chileno y el pueblo mapuche. En este sentido, el plan de argumentación sigue las dos dinámicas de la internacionalización, el primer momento correspondiendo a la dinámica nacional de proyección internacional y el segundo, a la dinámica internacional de penetración nacional.

\section{Marco metodológico}

Este trabajo, desde un enfoque comprensivo y explicativo busca, en primer lugar, entender los procesos de construcción de una identidad y el sentido que los actores otorgan a esa última y, en segundo lugar, intenta determinar las razones históricas y contextuales que dan origen a los procesos sociales estudiados. De acuerdo con lo anterior, yen el marco de mi tesis L'internationalisation d'un peuple autochtone: le cas des Mapuche et de la revendication d'une nation (La internacionalización de un pueblo indígena: el caso de los mapuche y de la reivindicación de una nación) en relaciones internacionales ${ }^{3}$, se entrevistó a un total de treinta y tres personas, mayoritariamente intelectuales mapuche y personas exiliadas en Europa, pero también funcionarios del Ministerio de Asuntos Exteriores chileno (MINREL), lugar en el que además se realizó una observación parti- 
cipante ${ }^{4}$. Por otra parte, se complementó esta investigación mediante la revisión de archivos e informes de organizaciones internacionales.

Al nivel epistemológico, la perspectiva constructivista que se adopta en el estudio de la transformación identitaria de los mapuche no se reduce solo al plano de las ideas, sino que toma en consideración que la constitución de una identidad también está sujeta a las luchas sociales (Cuche, 2016). La identidad, como forma de conocimiento social sobre un grupo, es un producto social y un factor de cambio social que entra en una relación dialéctica con la base social, tal como lo desarrollan Peter Berger y Thomas Luckmann (1966). Así, lo que es construido no es el objeto estudiado o sus condiciones materiales, sino que la idea del objeto, es decir, en nuestro caso, la identidad. Finalmente, tal y como explica Benedict Anderson (2006), en su libro sobre el origen del nacionalismo y la formación de comunidades imaginadas, el hecho de que estas últimas se construyen a partir de las ideas que circulan no significa que el objeto, es decir la comunidad, sea ficticia o irreal. En nuestro caso, la internacionalización de los mapuche participa plenamente de esa transformación identitaria tanto gracias a una transformación discursiva como de sus prácticas.

\section{La internacionalización de un pueblo indígena en exilio}

\section{Las primeras formas del exilio}

El exilio de los mapuche no empieza con el golpe de 1973, sino que, con los diversos desplazamientos internos o migraciones desde el campo a la ciudad, fundamentalmente a partir de la "pacificación de la Araucanía", y que se pueden calificar de exilio dado que los mapuche migran más allá del río Bío-Bío, considerado como la frontera entre el Estado chileno y el territorio mapuche antes de la invasión militar de 1860 a 1883. Esos movimientos son el origen de una profunda modificación de la autodefinición del pueblo mapuche, dado que los individuos que migran intentan disimular su origen para no ser discriminados. Sin embargo, su manera de hablar o su apariencia física son cuestiones suficientes para ser sujetos recurrentes de prácticas racistas.

Esta historia la podemos observaren la trayectoria de María, que Héctor Nahuelpán desarrolla en su artículo titulado Las 'zonas grises' de las historias mapuche (2013). Es la historia personal de una sirviente en casa de una familia burguesa, primero en Temuco y después en Santiago, donde se advierte la típica internalización del colonialismo, que tal y como lo explica Héctor Nahuelpán, corresponde al proceso en el cual "los modos y lógicas de poder donde la representación de lo mapuche como raza inferior, o como sujeto minorizado, transforma la violencia y el tutelaje como principales formas de integración-exclusión y de gobierno" (Nahuelpán Moreno, 2013, p. 15). Entonces, en la ciudad, los mapuche se vuelven en lo que se puede calificar de "diáspora invisible” (Aravena, 2001).

Sin embargo, el activismo político mapuche no desaparece y sigue vigente con la creación, al principio del siglo XX, de organizaciones mapuche, como la Sociedad Caupolicán Defensora de la Araucanía o la Federación Araucana de Manuel Aburto Panguilef, que comienzan a participar en el debate sobre la integración de los mapuche en la sociedad chilena (Bengoa, 2000). Este involucramiento político aumenta durante la reforma agraria, pues los mapuche se tornan protagonistas luego de la ocupación de varios fundos que reivindican como parte de sus tierras ancestrales. En algunos casos reciben la ayuda del Movimiento Campesino Revolucionario (MCR), vinculado al Movimiento de Izquierda Revolucionaria (MIR). Sin embargo, comienza a germinar una cierta autonomía mapuche, ya que algunas ocupaciones se ejecutan sin partidos políticos y son precisamente las organizaciones mapuche las que encabezan la iniciativa del proyecto de la ley indígena de 1972.

\section{El exilio en Europa y la formación del Comité Exterior Mapuche}

Como consecuencia del golpe militar de 1973, alrededor de cincuenta mapuche se distribuyen en Francia, Alemania, Bélgica, Suecia, Suiza y los Países Bajos entre 1974 y 1978. Arauco Chihuailaf (2005), exiliado en Francia, realizó ese conteo y él mismo reconoce que en realidad el número de exiliados mapuche debe haber sido mayor. Alejados de la sociedad chilena, los mapuche reafirman su identidad a medida que dejan de experimentar el racismo ejercido por esta. Tal es el caso de la experiencia del exilio de Rosario Railaf, quien produc- 
to de la militancia de su padre en el MCR, llega a los Países Bajos desde Lautaro, y narra que los holandeses no hacían la distinción entre quien era chileno y quien era mapuche (entrevista, 6 de enero 2018). Sin embargo, eso no significa que los mapuche dejaron de experimentar formas de racismo en Europa, solo subraya el hecho que un cambio de contexto social puede conducir a formas relativas de liberación. A esa razón social para explicar la afirmación identitaria de los mapuche, se suman razones políticas que provocan un distanciamiento con los partidos políticos de la izquierda chilena. La diáspora mapuche que se forma en Europa se puede así distinguir por los tres criterios definidos por Gabriel Sheffer (1986):

- El mantenimiento y el desarrollo de una identidad colectiva propia dentro del grupo "diasporizado";

- La existencia de una organización interna distinta a las existentes en el país de origen o en el "país de acogimiento";

• La presencia de vínculos fuertes con la "tierra de origen" (homeland).

La diáspora mapuche en Europa empieza a organizarse con la reunión de un grupo de mapuche que se realiza en Londres en enero 1978. A la iniciativa de Vicente Mariqueo, un ex dirigente estudiantil mapuche destacado durante los años 1960 y 1970, se suman 25 mapuche procedentes de distintas partes de Europa, y un único winka (no-mapuche), el exministro de agricultura de Salvador Allende, Jacques Chonchol. En un primer momento de la reunión, los participantes relatan su proceso de exilio, destacando la dificultad de pasar directamente desde el campo a la ciudad europea

En un segundo momento, los participantes del encuentro constatan que los mapuche desaparecidos o asesinados están ausentes de las listas hechas por los partidos políticos de izquierda. Aunque varios de ellos son miembros de partidos políticos chilenos de izquierda, la decisión de reunirse como mapuche surge, entre otras cosas, de esa voluntad de terminar con la falta de reconocimiento por parte de los partidos. Así la declaración de Londres, que resume el posicionamiento de los exiliados mapuche presentes, afirma que "el problema mapuche no puede continuar siendo un asunto secundario”(Comité Exterior Mapuche, 1978a). Además de lo señalado, la declaración formula dos objetivos que son, primero, dar a conocer en Europa la cultura de los mapuche y su realidad bajo la dictadura, y, segundo, generar redes de solidaridad para volver efectivo el primero. Al final, se busca informar y solidarizar (Arauco Chihuailaf, entrevista, 23 de febrero 2018). Así nace formalmente a partir de ese encuentro el Comité Exterior Mapuche.

\section{El activismo de la diáspora en contra de la dictadura}

El activismo del CEM consiste primero en la movilización contra la dictadura desde el exterior. La dispersión se vuelve de esa manera en un recurso político, dado que permite el activismo en diversos espacios nacionales (Ma Mung, 2004) y prescindir de la represión de la dictadura. Una forma de identidad imaginaria se desarrolla gracias al activismo que produce códigos comunes (Sökefeld, 2006). Esa posibilidad de ser multisituado permite al CEM recibir el apoyo de varias ONG, algunas con un estatuto consultivo en la ONU como, por ejemplo, Minority Group International o Survival International (Comité Exterior Mapuche, 1978b). El Comité crea, por otra parte, varios periódicos, incluido el Boletín Informativo Mapuche entre 1978 y 1982 y después la revista Huerrquen entre 1982 y 1984 (Chihuailaf, 2005). Los dos objetivos de la Declaración de Londres ya se cumplen con estos dos elementos.

A partir de la publicación del Decreto Ley n².568 de la dictadura en 1979, que apunta a la división de las comunidades mapuche, el CEM publica una crítica tajante a este en Le Monde diplomatique de Francia (Comité Exterior Mapuche, 1979). Esa tribuna provoca una reacción virulenta por parte del agregado cultural de la embajada de Chile que el mismo periódico acepta publicar. En su artículo, el CEM habla de "exterminación legal” del pueblo mapuche. El contenido de la reacción del agregado cultural a esa expresión señala de manera irónica que el CEM realiza "un pequeño análisis lleno de errores y que utiliza métodos marxistas más que manipulados y más que desusados” (“Diffamation de la junte chilienne”, 1979, traducción propia). Lo anterior demuestra entonces, la capacidad de perjuicio hacia las autoridades que logran los mapuche en la época. El CEM también genera conexiones con la organización Admapu, que se crea en 1978 en Chile para contribuir a la lucha contra el Decreto Ley n².568. En una entrevista con la revista Huerrquen en 1982, Melillan Painemal, uno de los dirigentes de Admapu, que viaja a Europa por una reunión del Consejo Mundial 
de los Pueblos Indígenas, en el cual fue electo para ocupar un puesto ejecutivo, subraya la importancia para los mapuche de poder expresarse sin intermediarios. Así, la distanciación hacia los partidos se hace cada vez más evidente.

La diáspora mapuche, a finales de los años setenta, empieza a utilizar las categorías de "etnia” y de "nación” para autodefinirse en un contexto de emergencia de los pueblos indígenas a nivel internacional y del movimiento de las minorías nacionales en Europa. Este último, calificado como nacionalitario, se expande durante la misma década en Europa y defiende el principio de las nacionalidades según el cual el Estado y la nación deben coincidir políticamente (Kernalegenn, 2013). Los vínculos con Admapu y la participación de Melillan Painemal en el Consejo Mundial de los Pueblos Indígenas inserta al CEM en el movimiento internacional de los pueblos indígenas, al mismo tiempo que se organizan tareas de información sobre los mapuche en el País Vasco, lo que permite generar vínculos de solidaridad y así concretar el nexo entre el CEM y el movimiento nacionalitario (Chihuailaf, 2005). La vinculación entre los dos movimientos gracias a las acciones del CEM subraya que ambos tienen demandas muy similares con la única distinción que el último no se enmarca en el contexto postcolonial. Así, el cambio de autoidentificación que ocurre en esa década tiene directa relación con el contexto en el cual el CEM se inserta y las conexiones que establece.

El cambio se percibe en 1985 cuando el CEM reivindica la autodeterminación como derecho inalienable (Comité Exterior Mapuche, 1985). Esa reivindicación es reafirmada, aunque bajo el término de "autonomía”, en una carta que el CEM envía a la organización Admapu para su quinto congreso, donde expresa lo siguiente:

"que la discusión avance en la profundización de este principio fundamental y que su importancia sea más claramente subrayada, porque pone de relieve la dimensión étnico-cultural de nuestras reivindicaciones y de nuestra lucha y porque tiene una implicación política nacional”(Comité Exterior Mapuche, 1987).

\section{La movilización en las organizaciones internacionales}

A finales de los años ochenta y con la transición democrática en Chile, el CEM comienza a desaparecer, lo que da lugar a un periodo de involucramiento en las organizaciones internacionales de parte de los mapuche que no volvieron a Chile. De esta manera, los mapuche forman parte del movimiento mundial de movilización de los pueblos indígenas, que le conduce, en algunas ocasiones, a encuadrar (Benford y Snow, 2000) su discurso en función del vocabulario de los derechos humanos, lo cual les permite tener acceso a las instancias de la ONU y, así, favorecer el cumplimiento de sus reivindicaciones. En dicho movimiento mundial, el concepto de "etnia” cae en desuso y la expresión de "pueblo indígena” se vuelve más utilizada ya que empieza a ser más codificada, sobre todo gracias al informe de José Martínez Cobo pedido por el Consejo Económico y Social de las Naciones Unidas (ECOSOC) y publicado en 1981. La categoría de "pueblo indígena" conduce a consagrar un derecho a la autodeterminación dado las disposiciones del derecho internacional, sobre todo de los Pactos internacionales de 1966 sobre derechos económicos, sociales y culturales y sobre derechos civiles y políticos, que, en sus artículos primero, afirma: “todos los pueblos tienen el derecho de libre determinación”.

A partir de 1990, se puede contabilizar más de cuarenta intervenciones por parte de los mapuche en la ONU, sobre todo en el Consejo de Derechos Humanos. Sin embargo, esas intervenciones son constreñidas por las reglas de presentación de la ONU. Así, no pueden superar algunos minutos y tienen que adoptar un lenguaje bastante jurídico para denunciar lo que se consideran como violaciones de los derechos humanos por parte del Estado chileno. Categorías tradicionales mapuche son utilizadas por la gente que interviene en el Consejo de Derechos Humanos, como werken, machi, o apo werken que es traducido como embajador (Mariqueo, 2017). Estas categorías tienen una doble función: primero, protegerse de la crítica, que le pueden hacer militantes involucrados en luchas locales, como carecer de legitimidad para hablar en nombre de todo los mapuche y, segundo, permite un uso transgresivo de los códigos de la ONU dado que el mapudungun no es reconocido como un idioma oficial por la organización.

La diplomacia de los mapuche es apoyada por la diáspora mapuche en Europa, la cual ayuda, por ejemplo, a constituir una "red internacional de apoyo al pueblo nación mapuche" que deposita regularmente peticio- 
nes en las embajadas chilenas de Europa ("Red Internacional de apoyo al Pueblo Nación Mapuche”, 2017). Este apoyo les permite también realizar gira en Europa para entregar una carta al Papa, cuestión que se hizo el 25 de octubre 2017 por parte de un grupo de mapuche de Chile y Argentina previa visita del Papa a Chile. Por otra parte, la diáspora solicita a los diputados europeos, como, por ejemplo, la española Estefanía Torres, para enunciar críticas de las políticas chilenas hacia los mapuche desde el Parlamento Europeo (Banchon, 2017).

A partir del año 2012, se crea la Comisión Mapuche de Derechos Humanos, cuya descripción, en el sitio web de la Misión Permanente Mapuche ante las Naciones Unidas, la asocia a esa última. El equipo de la Misión Permanente Mapuche se compone de diez personas, cada una con un puesto de consejero en un área específica (“Equipo de la Misión Mapuche ante la ONU”, 2015). Sin embargo, esa institución no tiene ninguna base legal, solo se apoya en su reivindicación de derecho a la autodeterminación. Pese a esa falta de reconocimiento oficial, la misión trata de igualar una representación diplomática oficial, como lo demuestra la denuncia del rechazo hecho por Suiza a Flor Calfuneo Paillalef respecto a concederle asilo político, tras la persecución que padeció en Chile por parte de las autoridades oficiales. Ese rechazo se funda en la cualificación de ella como la única embajada mapuche permanente en Suiza por parte de los miembros de la misión (Calfunao Paillalef y Mariqueo, 2017). Aunque ese argumento no tiene ninguna base jurídica, su formulación comprueba una vez más la voluntad de igualar a una representación diplomática oficial.

Para poder realizar intervenciones regulares, la Misión Permanente Mapuche se apoya, principalmente a través de Reynaldo Mariqueo, en la ONG Auspice Stella la cual tiene vínculos el autoproclamado Reino de la Araucanía $^{5}$, que se fundó en 1860 por el explorador francés ${ }^{6}$ Orélie-Antoine de Tounens y duró dos años hasta la expedición militar del Estado chileno hacia el sur. Al respecto, Carlos Contreras Painemal señala que, la alianza que establecen los mapuche con el denominado Reino es “táctica y no estratégicas” (Entrevista telefónica, 30 de enero 2018), es decir, que no tiene solo un carácter de adhesión ideológica sino también instrumental. Sin embargo, esa afirmación no pudo ser comprobada ante otros participantes de la ONG. Otras ONG abren también espacio a los mapuche no vinculados al Reino para intervenir en la ONU, como France Libertés o la Fundación Frantz Fanon.

\section{El Estado chileno frente a un nuevo actor diplomático}

En la movilización internacional, los mapuche entran en interacción con los representantes del Estado chileno. Estudiando los debates sobre la redacción de la Declaración de los Derechos de los Pueblos Indígenas de 2007, se constata que el Estado chileno es muy reticente a aceptar la noción de autodeterminación, intentando incluso de calificarla como "interna", es decir sin posible consecuencia de secesión. "Los pueblos indígenas pueden ser 'distintos y específicos' pero también desarrollarse como 'parte integral' de la nación chilena” resume Russel Lawrence Barsh (1996, p. 797, traducción propia).

El Estado chileno trata, por otra parte, de mostrar una imagen progresista de su política a nivel internacional. Se puede comprobar ello con los procedimientos del Examen Periódico Universal (EPU) que, desde 2006, realiza el Consejo de Derechos Humanos y que consiste en hacer una supervisión de la situación de los derechos humanos de los Estados miembros de la ONU. Chile pasa por ese procedimiento en 2009 y 2014. En el EPU de 2014, el Estado chileno responde a las numerosas recomendaciones que son hechas sobre los pueblos indígenas poniendo el énfasis en los resultados positivos de su política de diálogo y el carácter infundado de las críticas de violaciones de los derechos humanos (Consejo de Derechos Humanos, Naciones Unidas, 2014). Sin embargo, las críticas hechas al Estado chilenos son recurrentes en las intervenciones o los informes de las ONG que apoyan a los mapuche, entre ellas la Organización de Naciones y Pueblos No Representados (UNPO).

Además de esa postura ejemplar que el Estado defiende en las instancias de la ONU, aunque con una importante reticencia de avanzar en materia jurídica internacional respecto a los pueblos indígenas, una observación participante hecha por el autor de este texto entre el 19 de junio y el 14 de julio 2017 en el Ministerio de Asuntos Exteriores chileno (MINREL) corrobora la poca importancia institucional que el Ministerio dedica a esa movilización internacional. Los pueblos indígenas son un asunto que depende de la Unidad de Asuntos Indígenas, la cual se compone de una sola persona dentro de la Dirección de Derechos Humanos. El mismo discurso de ejemplaridad es dado por parte de los funcionarios que trabajan en el Ministerio sobre el avance del 
Estado chileno en materia de políticas indígenas. Además de eso, cuando se pregunta por la movilización mapuche a nivel internacional, todo conflicto es negado. Así, la preocupación de no dañar la postura progresista que el Estado chileno tiene a nivel internacional es tachada por esa observación.

En la movilización internacional se desarrolla de manera más apremiante el referente de identificación de "pueblo indígena" dado que lleva consigo varios derechos reconocidos por el derecho internacional. Sin embargo, el referente de nación viene a completar esa identificación de "ser mapuche", tal y como lo demuestra el uso de la expresión de "nación mapuche” que hace la Misión Permanente Mapuche ante las Naciones Unidas. Ser "nación" permite de este modo reivindicar una relación de igualdad a nivel internacional entre los mapuche y el Estado chileno. Esta relación tiene ahora que estudiarse en el espacio nacional mismo.

\section{Entre pueblo indígena y nación: la ambivalencia de los mapuche en el espacio nacional}

\section{La autonomización del movimiento mapuche}

Durante la transición democrática en Chile, a partir del año 1989, el concepto de autodeterminación se convierte en un tópico central en las reivindicaciones de los mapuche. Promesas de reconocimiento constitucional son hechas por el gobierno en el Acuerdo de Nueva Imperial firmado el 1 de diciembre de 1989. Sin embargo, la falta de reconocimiento del gobierno, la no inclusión de los pueblos indígenas en la Constitución, y la decepción de los mapuche respecto la ley indígena de 1993 (Figueroa, 2014) son factores que aceleran el proceso de autonomización de algunos grupos mapuche respecto el Estado chileno, como es el caso el Consejo de Todas las Tierras (CTT), creado en 1990. La figura del líder de esa organización, Aucán Huilcamán, es clave para entender la naturaleza de las reivindicaciones del CTT y su distanciamiento con otras organizaciones mapuche que participaron del Acuerdo de Nueva Imperial, por ejemplo, Admapu.

La trayectoria de Aucán Huilcamán es fuertemente internacionalizada. En la fundación del CTT en 1990, Aucán Huilcamán se presenta como el encargado de relaciones internacionales de la organización, aunque ya en 1987 viajó a la ONU en Ginebra como representante de Admapu (Martínez Neira, 2009). Luego, Aucán Huilcamán continua con su labor diplomática gracias a un pasaporte de la ONU que le permite viajar con inmunidad por América Latina, por su vínculo con la temática del "resarcimiento e indemnización a las víctimas de violaciones a los derechos humanos en pueblos indígenas” (Huilcamán, 2013). Dicha trayectoria explica en parte el manejo por el CTT de conceptos que se iban desarrollando a nivel internacional, como lo es la autodeterminación.

Por otra parte, el CTT promueve una vuelta a las autoridades tradicionales y, en ciertos aspectos de su discurso, manifiesta una invención de la tradición que trata de establecer una continuidad entre el pasado y el presente (Hobsbawm y Ranger, 2010). Es el CTT que da origen a la bandera mapuche, el Wenufoye, en 1992. En ese contexto, el referente de identificación de "pueblo indígena" es esencial para hacer valer el derecho de autodeterminación y reivindicar una continuidad cultural con el periodo precolonial. Acciones de recuperación de las tierras usurpadas son emprendidas, por ejemplo, con la toma del fundo Santa Clara el 10 de octubre 1989 (Pairican Padilla, 2012). El proceso de las tomas va aumentado durante los años, en paralelo al proceso de radicalización.

\section{La radicalización del movimiento mapuche y la demanda de una "liberación nacional”}

El proceso de radicalización del movimiento mapuche se cristaliza con el incendio de los camiones de compañías forestales en Lumaco en 1997 cuya consecuencia es la creación de la Coordinadora Arauco-Malleco (CAM). Los eventos de Lumaco representan, según Fernando Pairican (2013), un salto cualitativo en el movimiento, dado que a partir de ese momento se reivindica la devolución del territorio, y no solamente de las tierras, y se clarifica ideológicamente la "cuestión nacional mapuche”. Así, la CAM introduce una nueva expresión que es la de "liberación nacional” y que apunta a "reconstruir la nación mapuche” (Llaitul y Arrate, 2012). Esa reconstrucción se apoya en la coordinación entre varias comunidades. 
Una nueva figura tradicional es promovida por la CAM a partir de la traducción en mapudungun de guerrero, es decir el weichafe. Según Llaitul, esa figura se asocia a una ética de la acción política cuyo uso de la fuerza se hace solamente de manera defensiva. Así, se ve que el desarrollo ideológico de la CAM abarca todos los aspectos de una lucha política, del proyecto político a las acciones individuales. El referente de identificación dominante en ese marco ideológico es el de "nación", tal y como lo deja explicito el lema "liberación nacional mapuche” (Coordinadora Malleco Arauco, 2002).

La forma de organización de la CAM y su corpus ideológico se debe también a la trayectoria de su líder que contrasta con la de Aucán Huilcamán. Durante su juventud en la Universidad Católica de Valparaíso, Héctor Llaitul participa desde 1988 en el Frente Patriótico Manuel Rodríguez. Cuenta que, durante ese periodo, mezcla lecturas sobre marxismo y tercermundismo con acciones militantes en Valparaíso. A partir de los años 1990, Llaitul explica que empieza a "mapuchisarse" (Llaitul y Arrate, 2012). Una forma de reconstrucción identitaria está en proceso y se confirma cuando Llaitul afirma que puede reinterpretar su infancia como llena de códigos de la cultura mapuche (Llaitul y Arrate, 2012).

La trayectoria de Llaitul toma elementos de la teoría del foco desarrollada por Ernesto Guevara. La clandestinidad en la cual la CAM entra a principio de los años 2000 ante la represión estatal, se asocia a la teoría del foco y en que predomine como referente el concepto "nación" y el lema "liberación nacional”, que era una expresión dominante en el tercermundismo de los años sesenta, y que hoy se puede encontrar en el marco ideológico de los kurdos y de los palestinos con los cuales Héctor Llaitul estima que los mapuche tienen que solidarizarse (Llaitul y Arrate, 2012). Así las dos principales organizaciones mapuche del periodo posterior a la transición democrática tienen una semejanza en las trayectorias de sus líderes en cuanto al uso de referencia internacionales para el desarrollo de su marco ideológico. Sin embargo, se distinguen en cuanto al referente de identificación dominante en sus discursos y prácticas, el CTT remite más al de "pueblo indígena”, mientras que la CAM al de "nación".

\section{Una identificación plural que mezcla los espacios nacionales e internacionales}

Como se ve en la construcción de las organizaciones del CTT y de la CAM, existe una conexión con lo internacional ya sea a través de una movilización en las organizaciones internacionales, como por la reivindicación de una solidaridad con otros pueblos que son considerados iguales en cuanto a la lucha que tienen que emprender. Así, la identidad mapuche se construye con elementos que hace de la esfera internacional un factor mayor en la adopción de los referentes de identificación de "pueblo indígena” y de "nación”. La ambivalencia que existe entre "pueblo indígena" y "nación" no solo tiene una consecuencia nominativa, sino que se enmarca en prácticas y discursos políticos que tiene un efecto sobre la definición de la relación entre los mapuche y el Estado de Chile.

Mientras que el referente de "pueblo indígena" tenía una connotación reivindicativa fuerte cuando se creó el CTT, sobre todo con el surgimiento del concepto de autodeterminación, a partir de la ley indígena de 1993, ser indígena comienza también a ser parte de una política de Estado que tiene el poder de autentificar y legitimar ciertos procedimientos que, en última instancia, pueden decir quien es indígena y quien no lo es (Boccara, 2012). Por su parte, según el principio de subsidiariedad, que consagra la constitución chilena de 1980, los pueblos indígenas son considerados como actores de su propio desarrollo y pueden contribuir al desarrollo del país en general. Se le niega un poder decisorio, como lo demuestra el mandato que tienen los representantes indígenas de la Corporación Nacional de Desarrollo Indígena (CONADI).

Así, todo ese movimiento institucional evoluciona bajo la voluntad de implementar lo que corresponde a una versión neoliberal del multiculturalismo. Lo que se valora en "el indígena" es su cultura que puede ser objeto de desarrollo y así integrar al mercado. El etnoturismo participa de esa tendencia. En vez de contribuir a la etnicisación de diversos grupos, el multiculturalismo provoca más la etnificación, es decir la identificación, por parte de instituciones gubernamentales, de ciertos grupos como miembros de un pueblo indígena, debiendo así ser sujetos de las políticas respectivas (Boccara, 2012). El rechazo de participar en el Censo chileno de 2017 por parte de algunas comunidades, como la de Temucuicui, sobre todo en las zonas donde el conflicto es más agudo, refleja también una voluntad de no ser categorizados de tal forma y de pedir un reconocimiento substancial ("Rechazo de comuneros mapuche”, 2017). 
Sin embargo, la categoría de "indígena” puede también estar sujeta a otro tipo de comprensión, como el CTT lo demuestra. El CTT está también en una posición intermedia dado que el referente de "nación” es reivindicado por Aucán Huilcamán, aunque algunos intelectuales mapuche, como José Marimán (entrevista telefónica, 29 de enero 2018), consideran que el militante no desarrolla las implicancias teóricas que la noción incluye. Ese desarrollo teórico es emprendido por el Centro de estudios y de Documentación Mapuche Liwen.

\section{La creación de Liwen y el fracaso de Wallmapuwen}

Creado en Temuco en 1989, el Centro de Estudios y de Documentación Mapuche Liwen busca reunir documentos sobre la historia del movimiento mapuche, además de reflexionar sobre las formas políticas del proyecto político mapuche contemporáneo. La constitución de este centro se debe en parte a las conexiones internacionales que se establecieron entre militantes mapuche de Chile y militantes exiliados en Europa. El financiamiento de Liwen es apoyado por la Fundación suiza Charles Léopold Mayer para el Progreso del Ser Humano. El vínculo entre la Fundación y Liwen se establece gracias al militante chileno exiliado en Francia, Claudio Cratchley. Después de un viaje realizado con Alain Labrousse en 1987-1988 para rodar un documental sobre los mapuche, Claudio Cratchley presenta ese documental a la Fundación la cual acepta de financiar un proyecto para los mapuche y que será el proyecto de Liwen (Claudio Cratchley, entrevista, 9 de abril 2018).

El vínculo entre Liwen y Claudio Cratchley no es solamente en términos pecuniarios, sino que el militante exiliado aporta también una tradición ideológica que es la del estudio de las nacionalidades por los pensadores marxistas austriacos del siglo XIX, es decir Otto Bauer y Karl Renner. Esa línea de trabajo se complementa con los estudios hechos por los antropólogos latinoamericanos de la época, especialmente mexicanos como Guillermo Bonfil Batalla (Pedro Marimán, entrevista, 11 de febrero 2018). Así, el texto que publica José Marimán en 1990 "Cuestión mapuche, descentralización del Estado y autonomía regional” expresa en qué términos ideológicos Liwen se quiere enmarcar, y de esa manera, hacer avanzar la lucha mapuche.

En este texto, José Marimán critica un posible repliegue cultural por parte de los mapuche, lo que cualifica de ilusión política. Para poner fin a la "condición de minoría nacional oprimida del pueblo mapuche”, es necesario poder revitalizarla lengua mapuche en un espacio regional, sin tratar de conservar intacta una forma de cultura mapuche que sería entendida solo bajo un prisma folclórica (J. Marimán, 1992). La propuesta política de Marimán es una autonomía regional, asociada a la autodeterminación de un territorio dado, que debería ser realizado por los mapuche a través de la creación de una asamblea cuya contribución sería, de esa manera, una profundización democrática de la sociedad en su conjunto.

El marco ideológico de Marimán le conduce a sostener que el movimiento mapuche se convierte en un movimiento nacionalitario, es decir, que defiende las nacionalidades sin necesariamente reivindicar la formación de un Estado-nación correspondiente a esa nacionalidad. Para desarrollar esta idea, Marimán se apoya en Maxime Rodinson, pensador marxista francés, distinguiendo el termino de "nacionalitario" del principio de las nacionalidades y a su vez de la ideología del nacionalismo europeo, lo que contradice así la definición canónica del término. La síntesis entre diferentes pensamientos la realiza Marimán, apoyándose en el trabajo de Guillermo Bonfil Batalla, al rechazar el indianismo como categoría que entorpece el reforzamiento de la identidad mapuche dado la separación que opera entre la cultura, la lengua y la identidad de un pueblo (J. Marimán, 1992).

La creación del partido mapuche Wallmapuwen, que significa “ciudadanos del país mapuche”, en el año 2005 en Temuco, se inscribe en la continuidad de esa reflexión y el fracaso electoral que va a vivir lleva a reconsiderar el potencial movilizador de una reflexión nacionalitaria para el pueblo mapuche. Wallmapuwen define una estrategia política que se concentra en las elecciones municipales como condición para realizar un proyecto autonomista y nacionalitario (Naguil, 2005). En su Declaración de Principios, el primero principio enunciado demuestra su inscripción ideológica en el marco ideológica elaborado por José Marimán. Este señala así que, lo que une los mapuchees "una cultura, una misma historia, una lengua propia y nacional, y por sobre todo, la voluntad de desarrollar una comunidad de destino, es decir, una Nación” ("Declaración de Principios de Wallmapuwen”, 2005). 
Apoyándose en textos internacionales, sobre todo en los Pactos de 1966 pero no en el Convenio 169 de la OIT, Wallmapuwen defiende la autodeterminación sin considerarse como partido étnico dado su voluntad de no ser asociado a la "cuestión indígena campesina" y con la intención de tomar por ejemplo las Comunidades autónomas en España (“Quiénes somos - Wallmapuwen”, s. f.). El referente de "pueblo indígena” está también ausente en la Declaración de Principios. Las implicancias de esa ausencia se concretan en los acuerdos de cooperación de Wallmapuwen con partidos regionalistas europeos, entre ellos el partido Izquierda Republicana de Cataluña (ERC), el Bloque Nacionalista Galego (BNG) o con el partido político europeo la Alianza Libre Europea (ALE). En vez de involucrarse en las instituciones internacionales relativas a los pueblos indígenas, Wallmapuwen apunta a "una estrategia de relaciones de pueblo a pueblo, sin intermediarios de ninguna naturaleza. (...) Esto demuestra que la voz mapuche es demandada en el concierto internacional y eso debe provocarnos un gran orgullo como pueblo", explica Víctor Naguil, encargado de las relaciones políticas e internacionales del partido (Cayuqueo, 2008).

Sin embargo, Wallmapuwen es un fracaso electoral. Primero, no logran registrar el partido pues no cumplen los requisitos de inscripción, y aunque luego sí lo hacen, gracias a la reforma a la ley de partidos, no logran mantenerse como organización, dado su bajo número de militantes y teniendo así que retirarse de las listas oficiales de los partidos en 2017 (Gutiérrez y Galvez, 2017). Segundo, sus resultados son muy bajos. En las elecciones municipales de 2016, presenta un solo candidato que reúne $0,39 \%$ de votos a nivel nacional. Si existen varias razones que explican ese fracaso electoral, como la tradición de un voto de derecha en la Araucanía o la incompatibilidad entre la tradición política mapuche y la forma partidaria, otra interpretación, complementaria a los factores precedentes, puede también ser que la ausencia de un referente asociado al hecho de ser parte de un pueblo indígena contribuye a una falta de identificación de los mapuche con el partido. La creación de un movimiento nacionalitario mapuche sería así demasiado excluyente del referente de "pueblo indígena", lo cual estructura una parte del movimiento mapuche y sobre todo demuestra la falta de apoyo por parte de las comunidades al partido mapuche.

\section{La fragmentación de un movimiento político por la internacionalización}

Los usos que se pueden hacer de cada referente de identificación, el de "pueblo indígena” y de "nación”, dejando al margen la categoría de "etnia" dado que se confunde mayoritariamente con la de "nación" y que su uso es menor, se distinguen según los actores que se reivindican de cada referente en particular. Sin embargo, esa distinción tiene límites por el hecho que autodefinirse como "nación” o como "pueblo indígena” conduce a adoptar un cierto discurso y determinadas prácticas. Una ambivalencia identitaria se constituye entre los dos referentes movilizados por los mapuche dado que cada uno lleva el movimiento político por una dirección diferente que puede ser, por un lado, más asociado al multiculturalismo y, por el otro, a la implementación de una autonomía regional. Y esos dos referentes se construyeron a través de las dos dinámicas del proceso de internacionalización.

El resultado de esa construcción identitaria es una fragmentación de los referentes de identificación y, por ende, del movimiento mapuche, como lo demuestra la multitud de las organizaciones mapuche que existen y la diversidad de su marco ideológico. Así, además de la ambivalencia identitaria, se suma una segunda que está entre fragmentación e hibridación, esa última pudiendo definirse como una mezcla de discursos y prácticas que en apariencia no atestigua de la naturaleza diferente de los componentes de la mezcla. Eso significa que el uso que se hace por los mapuche de los dos referentes de identificación participa a la vez a la fragmentación del movimiento y a la hibridación de sus categorías discursivas, como se puede ver en los discursos del CTT y de la CAM donde la "nación mapuche" se mezcla al hecho de ser "pueblo indígena” a la vez que se distingue cada uno en el momento de asociarles otros conceptos. Esa situación es el resultado del proceso de internacionalización que, a través de sus dos dinámicas, se concretiza de varias formas como las del exilio, las conexiones entre diversos espacios nacionales, o la movilización en organizaciones internacionales.

La contradicción que podía existir entre la construcción identitaria, que defiende una pertenencia particular con el hecho de internacionalizarse, es decir participar del movimiento de globalización que implica desarrollar un sentido de pertenencia más global, encuentra una forma de resolución en el hecho que la internacionalización puede participar en la construcción de un referente de identificación que no se constituye- 
ron en la exclusión del otro sino que a través de conexiones entre diferentes actores y gracias a intercambios de contenido ideológico como de prácticas.

Ese intercambio está en el origen de una base común que, aunque haya una fragmentación, permite a la mayoría de los mapuche de tener un concepto que todos defienden más o menos sin que necesariamente todos entiendan lo mismo por él. Esa base común se encuentra en el concepto de autodeterminación, o sus coralarios.

"Lo que no existe en el pueblo mapuche, es esa coordinación. Cada uno levanta un tema, pero frente a los temas mayores no hay cuestionamiento. A veces, tenemos diferencias sobre la manera de abordar los temas territoriales, pero no para el discurso común”, confirma Iván Carilao (entrevista, 21 de diciembre 2017), representante mapuche en la CONADI y miembro fundador de la Identidad Territorial Lafkenche.

Sin embargo, ese mismo concepto de autodeterminación es sujeto a dos usos que lo hacen también ambivalente y que se asocian a los diferentes referentes de identificación. El primer uso es vinculado a la aplicación del Convenio 169 y particularmente a los artículos 7 y 8. La ratificación del Convenio por Chile se realiza en 2008, mientras que era una promesa del Acuerdo de Nueva Imperial de 1989. Su implementación tiene varias faltas, pues la adopción del primer decreto $\left(n^{\circ} 124\right)$ que viene a regular la aplicación del Convenio, aunque la Corte Suprema declara en 2000 que el Convenio es autoejecutable (Aylwin, Carmona, Silva, y Yáñez, 2009), no pasa por un proceso de consulta de los pueblos indígenas. El siguiente decreto $\left(n^{\circ} 66\right)$ define un proceso en cinco etapas para realizar la consulta lo que pide un tiempo importante de involucramiento y genera una necesidad de asesoramiento jurídico mayor. Ese proceso reduce así considerablemente el grado de autodeterminación, transformándolo en un procedimiento administrativo de larga duración. Por otra parte, la autodeterminación tiene una cara ilegal representada por las tomas de terreno que realizan varias comunidades o militantes de la CAM. El hecho de vivir en un terreno ocupado y en comunidad demuestra la co-constitución que existe entre prácticas y desarrollo ideológico en cuento al concepto de autodeterminación, dado que la mayoría de las organizaciones mapuche apoyan dicho movimiento.

\section{Conclusión}

Las dos dinámicas de la internacionalización, una nacional con proyección internacional y una internacional con penetración nacional, de los mapuche originan de un cambio identitario gracias a la variedad de referentes de identificación, especialmente los de "pueblo indígena” y de "nación”, a los cuales dan lugar. Ese cambio demuestra el carácter variante de la identidad que depende de contextos, prácticas y discursos diferentes. Se establece además que, el proceso de internacionalización conduce a una variación más grande al momento de autodefinirse dado que los actores son expuestos a varios lugares de movilización que tienen cada uno un marco ideológico, oportunidades y obligaciones distintos. Ser pueblo indígena en la ONU no implica lo mismo en términos de prácticas y de discursos que reivindicar el estatuto de minoría nacional y constituir alianzas con partidos europeos que se incluyen en la misma categoría.

La primera dinámica de internacionalización, que empieza con el exilio después del golpe de 1973, conduce a la formación de solidaridades entre Chile y Europa y a una movilización en contra de la dictadura desde el extranjero. Esa movilización participa de una producción ideológica que adopta conceptos como los de "etnia" y "nación" en parte gracias a sus intercambios con Admapu o por el contexto del movimiento nacionalitario al fin de los años 1970. Con la desaparición del CEM y la importancia que toma el movimiento indígena a nivel internacional, se crea en 2012 la Comisión Mapuche de Derechos Humanos que trata de enfrentar al Estado chileno en el terreno de la diplomacia multilateral, especialmente en el ámbito de los derechos humanos, lo que también viene a demostrar los límites de las instituciones consagradas a los pueblos indígenas a nivel internacional dado que no permiten siempre hacer frente a los Estados directamente. Así, la búsqueda de instaurar una relación de igualdad entre los mapuche y el Estado chileno es manifestada con esta iniciativa.

La segunda dinámica confirma la importación de elementos provenientes de la primera dinámica, como el concepto de autodeterminación. En el contexto de la transición democrática, el movimiento mapuche empieza a autonomizarse dado el poco beneficio que encuentra en la cooperación con las autoridades. Primero el 
CTT, y después de la CAM, son las organizaciones mapuche que redefinen el contenido conceptual de lo que es "ser mapuche", en parte gracias a las conexiones o las comparaciones internacionales de cada líder de las dos organizaciones. En ese contexto, los dos referentes de identificación de "pueblo indígena” y de "nación” se afirman. El trabajo del Centro de Estudios y de Documentación Liwen viene confirmar el referente de "nación”, demarcándose de una voluntad de restablecer autoridades tradicionales. El fracaso de la concretización política de ese trabajo ideológico, que es Wallmapuwen, conduce a la constatación de una fragmentación de los referentes de identificación movilizados por los diferentes actores mapuche. La fragmentación provocada por la internacionalización se inserta, sin embargo, en una ambivalencia cuyo segundo término es la hibridación como lo demuestra el hecho que algunos actores pueden mezclar los referentes sin encontrar mayor contradicción o tratando de superarlas. Si la internacionalización puede producir divisiones, ella provoca también mezclas de prácticas y de discursos que puede tener un valor productivo importante.

\section{Agradecimientos}

Agradezco a mi director de tesis Guillaume Devin por sus consejos durante todo el proceso de investigación. Agradezco también a Mafe por su amor y su infinita ayuda. Mis últimos agradecimientos son para la persona anónima que revisó este artículo y por sus valiosos comentarios.

\section{Notas}

\footnotetext{
1 “Mapuche” significando "gente de la tierra”, no se necesita poner una "s” al plural dado que este ya es contenido en el sustantivo.

${ }^{2}$ Organización que en los años 1930 dependía de la esfera soviética.

${ }^{3}$ Ese artículo se desprende de mi tesis para optar al grado de Magíster en Ciencia Política mención Relaciones Internacionales, dirigida por Guillaume Devin, y defendida el día 4 de junio de 2018 en Sciences Po, París.

${ }^{4}$ Entre el 19 de junio y el 14 de julio 2017. Cabe señalar que, algunos entrevistados han sido anonimizados previa solicitud de ellos.

${ }^{5}$ Es preciso señalar que, los mapuche nunca tuvieron una monarquía y que las actividades de estos representantes han sido ampliamente criticada por diferentes dirigentes mapuche, tal y como se aprecia a continuación: "Esto no solamente nos parece pésimo, sino que es una locura. Los mapuches jamás hemos tenido un rey ni un príncipe. Esta persona vino hace 20 años y nosotros lo echamos de nuestras tierras, y nuestros hermanos no tienen por qué reconocer ni recibir nada de él”, señaló José Santos Millao, líder y vocero de la organización Admapu. (Las Últimas Noticias, 13 de Julio de 2014). ${ }^{6}$ El estatuto de “explorador” remite a una figura histórica precisa estrechamente vinculada a la conquista colonial y que tiene por
meta“descubrir” tierras que no están bajo el dominio occidental. Orélie-Antoine de Tounens llega con ese propósito a Chile antes de
entrar en contacto con los mapuche.
}

\section{Bibliografía}

Anderson, B. (2006). Imagined Communities: Reflections on the Origin and Spread of Nationalism. Londres, UK: Verso.

Appadurai, A. (1996). Modernity At Large: Cultural Dimensions of Globalization. Minnesota, United States: University of Minnesota Press.

Aravena, A. (2001). La diáspora invisible. El Correo de la UNESCO, (9), 18-20. Recuperado de http:// unesdoc.unesco.org/images/0012/001235/123512s.pdf

Aylwin, J., Carmona, C., Silva, H., y Yáñez, N. (2009). Las implicancias de la ratificación del Convenio N¹69 de la OIT en Chile. Temuco, Chile: Observatorio Ciudadano.

Banchon, M. (2017, octubre 2). Desde Europa, la causa mapuche. DW.COM. Recuperado de http://www.dw.com/ es/desde-europa-la-causa-mapuche/a-40760390 
Barsh, R. L. (1996). Indigenous Peoples and the UN Commission on Human Rights: A Case of the Immovable Object and the Irresistible Force. Human Rights Quarterly, 18(4), 782-813. doi: 10.1353/hrq.1996.0038

Benford, R. D., y Snow, D. A. (2000). Framing Processes and Social Movements: An Overview and Assessment. Annual Review of Sociology, 26, 611-639. doi: 10.1146/annurev.soc.26.1.611

Bengoa, J. (2000). Historia del pueblo mapuche Santiago de Chile: Ediciones Sur.

Berger, P. L., y Luckmann, T. (1966). The Social Construction of Reality: A Treatise in the Sociology of Knowledge. New York, United States: Anchor Books.

Boccara, G. (2012). Multiculturalisme, néolibéralisme, démocratisation. En C. Gros y D. Dumoulin-Kervran (Eds.), Le multiculturalisme au concret?: Un modèle latino-américain?? Paris, France: Presse Sorbonne Nouvelle. Recuperado de http://books.openedition.org/psn/599

Calfunao Paillalef, F., y Mariqueo, R. (2017, enero 2). Comunicado: Misión Permanente Mapuche ante la Organización de Naciones Unidas. Recuperado 30 de marzo de 2018, de http://mision-nacion-mapuche.info/2013/ 08/15/comunicado-mision-permanente-mapuche-ante-la-organizacion-de-naciones-unidas/

Cayuqueo, P. (2008, febrero 7). Víctor Naguil, Encargado de Relaciones Políticas: Partido Wallmapuwen responde a «revelaciones» de El Mercurio. Recuperado 31 de enero de 2018, de https:/www.mapuche-nation.org/ espanol/html/noticias/ntcs-344.htm

Chihuailaf, A. (2005). Mapuche: gente de la tierra. Mas allá del Nuke Mapu (Madre Tierra), el exilio. Contribuciones desde Coatepec, (8), 157-171.

Comité Exterior Mapuche. (1978a). Declaración Encuentro Mapuche de Londres. Recuperado de http:// www.nacionmulticultural.unam.mx/movimientosindigenas/docs/36.pdf

(1978b). Primera Conferencia de los Mapuches Exiliados en Europa.

(1979, julio 1). Les Mapuches menacés d’extermination légale. Le Monde diplomatique. Recuperado de https://www.monde-diplomatique.fr/1979/07/A/35189

50-51.

(1980). A dos años del Primer Encuentro Mapuche de Londres. Boletín Informativo Mapuche, (7),

(1985). Comunicado.

(1987). Carta a Ad Mapu con motivo de su V Congreso.

Consejo de Derechos Humanos, Naciones Unidas. (2014). Informe del Grupo de Trabajo sobre el Examen Periódico Universal - Chile (No. A/HRC/26/5). Ginebra. Recuperado de https://documents-dds-ny.un.org/doc/ UNDOC/GEN/G14/129/00/PDF/G1412900.pdf?OpenElement

Coordinadora Malleco Arauco. (2002). El Pueblo Mapuche y su lucha de liberación. Recuperado 23 de abril de 2019 de http://www.archivochile.com/Pueblos_originarios/lucha/POlucha0010.pdf

Cuche, D. (2016). La notion de culture dans les sciences sociales. Paris, France: La Découverte.

Declaración de Principios de Wallmapuwen. (2005, diciembre). Recuperado 15 de diciembre de 2017, de http:// www.mapuche-nation.org/espanol/html/noticias/cmdo-216.htm

Devin, G. (2001). L’internationalisation de la société française. En Annuaire Français des Relations Internationales (pp. 317-321). Bruxelles, Belgique: Bruylant. 
Diffamation de la junte chilienne. (1979, agosto 1). Le Monde diplomatique. Recuperado de https://www.mondediplomatique.fr/1979/08/A/35221

Equipo de la Misión Mapuche ante la ONU. (2015). Recuperado 30 de marzo de 2018, de http://mision-nacionmapuche.info/nosotros/equipo-de-la-mision/

Figueroa, V. (2014). Formulación de políticas públicas indígenas en Chile: evidencias de un fracaso sostenido. Santiago de Chile: Editorial Universitaria.

Gutiérrez Chong, N., y Gálvez González, D. (2017). La cultura política en el pueblo mapuche: el caso Wallmapuwen. Revista Mexicana de Ciencias Políticas y Sociales, 62(231), 137-165. doi: 10.1016/S0185-1918(17)300417

Hobsbawm, E. J., \& Ranger, T. O. (Eds.). (2010). TheInvention of Tradition. Cambridge: Cambridge University Press.

Huilcamán, A. (2013, abril). “Antes de cinco años formaremos un autogobierno”. Recuperado 25 de septiembre de 2018, de http://www.revistanos.cl/2013/05/aucan-huilcamanantes-de-cinco-anos-formaremos-unautogobierno/

Kernalegenn, T. (2013). Le réveil des revendications régionalistes et nationalitaires au tournant des années 1968?: analyse d’une «?vague?» nationale. Fédéralisme Régionalisme. Recuperado de https:/popups.uliege.be:443/ 1374-3864/index.php?id=1195

La Araucanía: Rechazo de comuneros mapuche tensiona aplicación del Censo. (2017, abril 19). Recuperado 25 de septiembre de 2018, de https://www.cooperativa.cl/noticias/pais/poblacion/censos/la-araucania-rechazo-decomuneros-mapuche-tensiona-aplicacion-del-censo/2017-04-19/123504.html

Llaitul, H., y Arrate, J. (2012). Weichan: conversaciones con un weychafe en la prisión política. Santiago de Chile: Ceibo Ediciones.

Ma Mung, E. (2004). Dispersal as a Resource. Diaspora: A Journal of TransnationalStudies, 13(2), 211-225. doi: 10.1353/dsp.2008.0001

Mallon, F. E. (2011). Victims into Emblems: Images of the Ránquil Massacre in Chilean National Narratives, 1934-2004. Labor, 8(1), 29-55. doi: 10.1215/15476715-2010-044

Marimán, J. (1992). Cuestión mapuche, descentralización del Estado y autonomía regional. Caravelle, 59(1), 189205. doi: 10.3406/carav.1992.2522

Mariqueo, R. (2017). Presentación de Reynaldo Mariqueo en el 35º Período de Sesiones -Tema 3- del Consejo de Derechos Humanos de la ONU. Ginebra: Consejo de Derechos Humanos, Naciones Unidas. Recuperado de https://www.mapuche-nation.org/espanol/html/documentos/doc-151.html

Martínez Neira, C. (2009). Transición a la democracia, militancia y proyecto étnico. La fundación de la organización mapuche Consejo de Todas las Tierras (1978-1990). Estudios Sociológicos, 27(80), 595-618.

Naguil, V. (2005). Autogobierno en el País Mapuche-Parte II: Hacia la creación de un partido autonomista. Periódico Azkintuwe, (15), 12-14. Recuperado de https://www.mapuche-nation.org/espanol/html/articulos/art97.htm

Nahuelpán Moreno, H. (2013). Las “zonas grises” de las historias mapuche. Colonialismo internalizado, marginalidad y políticas de la memoria. Revista de Historia Social y de las Mentalidades, 17(1), 11-33. 
Pairican Padilla, F. (2012). Sembrando Ideología: el Aukiñ Wallmapu Ngulam en la Transición de Aylwin (19901994). SudHistoria: Revista Digital en Estudios desde el Sur, (4), 12-42.

F. (2013). Lumaco: La cristalización del movimiento autodeterministamapuche. Revista de Historia Social y de las Mentalidades, 17(1), 35-57.

PichinaoHuenchuleo, J. (2013). Los parlamentos hispano-Mapuche como escenario de negociación simbólico político durante la colonia. En P. Marimán, H. Nahuelpan Moreno, y H. Huinca Piutrin (Eds.), Ta iñfijkexiparakizuameluwun. Historia, colonialismo y resistancia desde el país mapuche (pp. 25-42). Temuco, Chile: Ediciones Comunidad de Historia Mapuche.

Quiénes somos - Wallmapuwen. (s. f.). Recuperado 26 de septiembre de 2018, de http://wallmapuwen.cl/quienessomos/

Red Internacional de apoyo al Pueblo Nación Mapuche en Chile manifiesta repudio ante asesinato a jóvenes mapuche Luis Marileo Cariqueo y Patricio González. (2017, junio 19). Recuperado 18 de enero de 2018, de http://www.mapuexpress.org/?p=18721

Sheffer, G. (Ed.). (1986). Modern Diasporas in International Politics. Londres: Croom Helm.

Sökefeld, M. (2006). Mobilizing in transnational space: a social movement approach to the formation of diaspora. Global Networks, 6(3), 265-284. doi: 10.1111/j.1471-0374.2006.00144.x 